\title{
PPPM in Cancer
}

\author{
"FIRST DO NO HARM" AND THE IMPORTANCE \\ OF PREDICTION IN ONCOLOGY \\ Characiejus $D^{1,2}$, Hodzic $J^{3}$, Jacobs $J J L^{3}$ \\ ${ }^{1}$ Faculty of Medicine, Vilnius University, M.K.Čiurlionio \\ 21, Vilnius, Lithuania \\ ${ }^{2}$ Centre for Innovative Medicine, Žygimantų 9, Vilnius, \\ Lithuania \\ ${ }^{3}$ VU Medical Centre, de Boelelaan 1117, Amsterdam, The \\ Netherlands
}

Present cancer treatment strategies are based on the assumption that a therapy may work ("response") or not work ("no-response"). In patients who do not respond to therapy, the course of disease is usually believed to be the same as without treatment. However, the existing evidence suggests that current cancer treatment modalities may also have a cancer-promoting effect. In this presentation, the data will be reviewed suggesting that tumour growth / metastatic spread can be stimulated and survival of patients in certain subgroups can be decreased by surgery, irradiation, chemotherapy and immunotherapy. Thus, results of cancer treatment may be improved by detecting and using biomarkers that correlate with positive or negative therapeutic effect. Small comparative trials based on such biomarkers rather than large phase III trials may be preferable for testing efficacy of new drugs. Ignoring biomarkers that correlate with positive or negative therapeutic effect may not be compatible with the ethical principle "First Do No Harm".

\section{THE USE OF VARIATIONS IN PROTEOMES TO PREDICT, PREVENT, AND PERSONALIZE TREATMENT FOR CLINICALLY NONFUNCTIONAL PITUITARY ADENOMAS}

Zhan X, Desiderio DM

Department of Neurology, University of Tennessee Health Science Center, 847 Monroe Avenue, Room 108, Memphis, TN 38120 USA

The traditional assessment of the level of one serumhormone is difficult to detect clinically nonfunctional (NF) pituitary adenomas; furthermore, no effective chemical therapy exists to treat an NF pituitary adenoma. An NF pituitary adenoma contains highly heterogeneous cell types [1] that are the distinguishing pathophysiological basis that causes the proteomic variation. In combination with multiple endogenous and exogenous factors, that proteomic variation is the basis for personalized patient treatment. A more accurate multiple-parameter global systematic strategy will replace the limited single-factor strategy in the prediction, prevention, and personalized treatment of NF pituitary adenomas. The global-proteomic strategy offers the promise for the multiple-parameter assessment for the predictive diagnosis, measurement of preventive and therapeutic response, and personalized patient treatment. The diagram of the use of proteomic variation to predict, prevent, and personalize treatment of NF adenomas is shown (Fig. 1). A tissue proteomic-variation study would lead to the accurate molecular classification of an NF adenoma that will contribute to identification of personalized patients. Modalities of the altered tissue-proteome of a personalized patient will realize the personalized treatment of NF pituitary adenomas. This therapeutic response will be assessed with body-fluid (serum and/or CSF) proteomic/ peptidomic variations to determine whether tumor progression is inhibited or halted. The high-risk population that might develop an NF pituitary adenoma will be determined with a predictive diagnosis strategy-body-fluid (serum and/or CSF) proteomic/peptidomic variations. Modalities of the altered tissue-proteome of the high-risk population will realize the interventional prevention. This preventive response will be assessed with body-fluid (serum and/or $\mathrm{CSF}$ ) proteomic/peptidomic variations to determine the preventive effects and whether tumor occurrence is halted. The serum/cerebrospinal fluid (CSF) proteomic/peptidomic variation would lead to serum/CSF protein/peptide pattern diagnostics, and the protein/antibody microarray for the predictive diagnosis and measurement of interventional prevention and personalized therapeutic response.

Proteomic variations offer numerous opportunities to predict, prevent, and provide a personalized treatment (PPPM) for NF pituitary adenomas. Currently, whole tissue-proteome variations (protein expression; hormone isoforms; modifications) are studied in NF pituitary adenomas. A series of pituitary adenoma proteomic data included 111 proteins identified from an NF 
pituitary adenoma tissue [3], 56 differentially expressed proteins from $11 \mathrm{NF}$ pituitary adenoma and eight control tissues (Fig. 2) [1], hormone isoforms (growth hormone and prolactin) (Fig. 3) [5], and nine nitroproteins and three nitroprotein-protein complexes from an NF pituitary adenoma tissue (Fig. 4) [6] and eight nitroproteins from a pituitary control tissue [7, 8]. Signaling pathway networks associated with NF pituitary adenomas are mined from those whole tissue-proteome variation data (Fig. 5) [9]. Four important pathway-network systems, including mitochondria dysfunction, oxidative stress, cell-cycle dysregulation, and MAPK-signal abnormality, for the NF adenoma are identified. The follow-up studies of those tissueproteome data would contribute to the accurate molecular classification for the diagnosis of personalized patients and their personalized treatment. Moreover, the complete tissueproteomic databases and body-fluid proteomic/peptidomic databases unique to single-cell type pituitary adenomas and different pathophysiological stages, must be accurately established, and the comprehensive computation model system needs to be developed, towards that PPPM goal.

\section{References:}

1. Moreno CS, Evans CO, Zhan X, Okor M, Desiderio DM, Oyesiku NM. Novel molecular signaling in human clinically non-functional pituitary adenomas identified by gene expression profiling aproteomic analyses. Cancer Res. 2005; 65: 10214-22.

2. Zhan X, Desiderio DM. The use of variations in proteomes to predict, prevent, and personalize treatment for clinically nonfunctional pituitary adenomas. EPMA J. 2010; 1: 439-59.

3. Zhan X, Desiderio DM. A reference map of a pituitary adenoma proteome. Proteomics 2003; 3: 699-713.

4. Zhan X, Desiderio DM. Comparative proteomics analysis of human pituitary adenomas: current status and future perspectives. Mass Spectrom Rev 2005; 24: 783-813.

5. Zhan X, Giorgianni F, Desiderio DM. The proteomics analysis of growth hormone isoforms in human pituitary. Proteomics 2005; 5: $1228-41$.

6. Zhan X, Desiderio DM. Nitroproteins from a human pituitary adenoma tissue discovered with a nitrotyrosine affinity column and tandem mass spectrometry. Anal Biochem 2006; 354: 27989.

7. Zhan X, Desiderio DM. The human pituitary nitroproteome: detection of nitrotyrosyl-proteins with two-dimensional Western blotting, and amino acid sequence determination with mass spectrometry. Biochem Biophys Res Commun 2004; 325: 1180 6.

8. Zhan X, Desiderio DM. Linear ion-trap mass spectrometric characterization of human pituitary nitrotyrosine-containing proteins. Int J Mass Spectrom 2007; 259: 96-104.

9. Zhan X, Desiderio DM. Signaling pathway networks mined from human pituitary adenoma proteomics data. BMC Med Genomics 2010; 3: 13

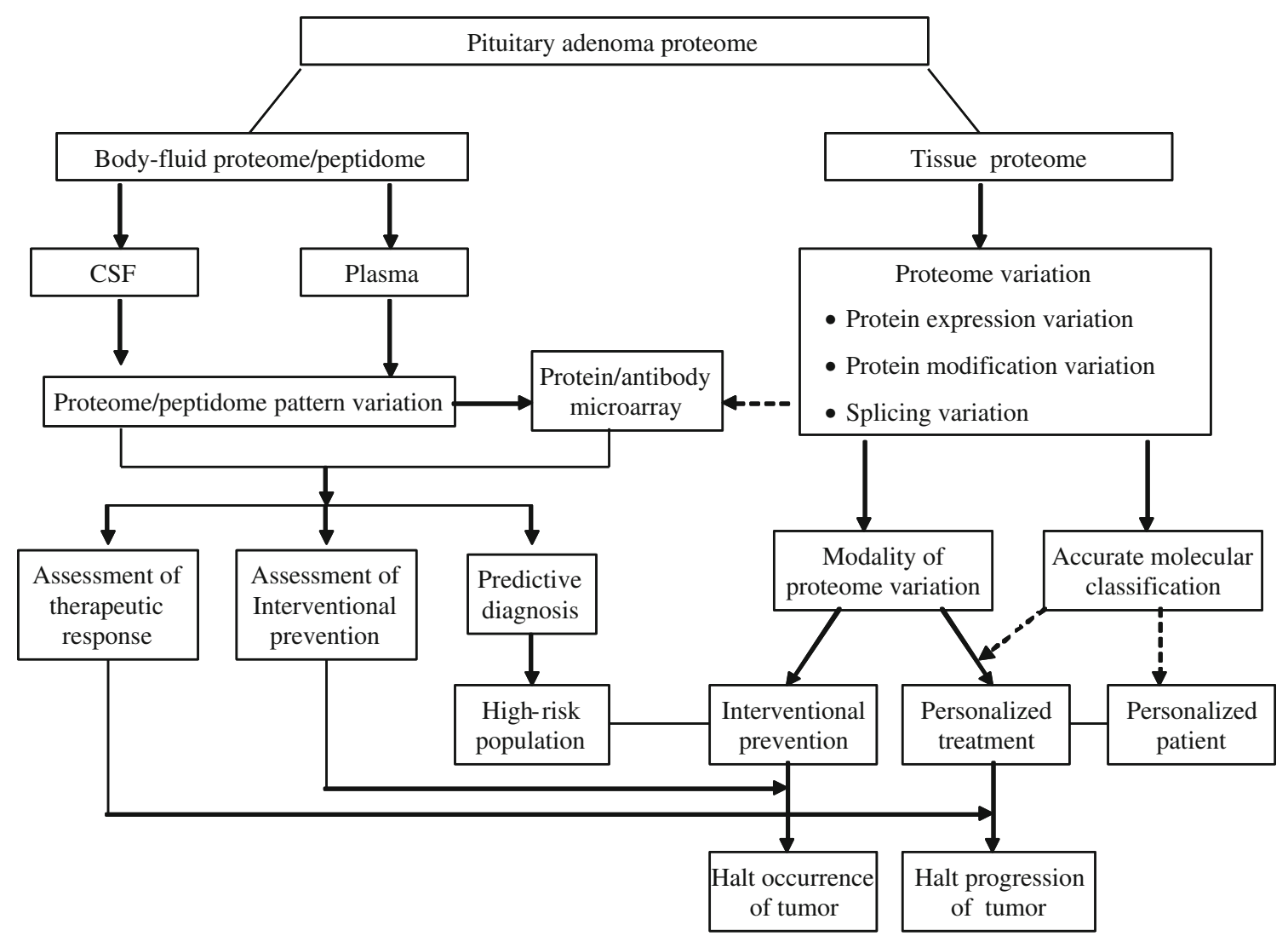

Fig. 1. Diagram of the use of proteomic variations to predict, prevent, and personalize treatment for NF pituitary adenomas [2] 

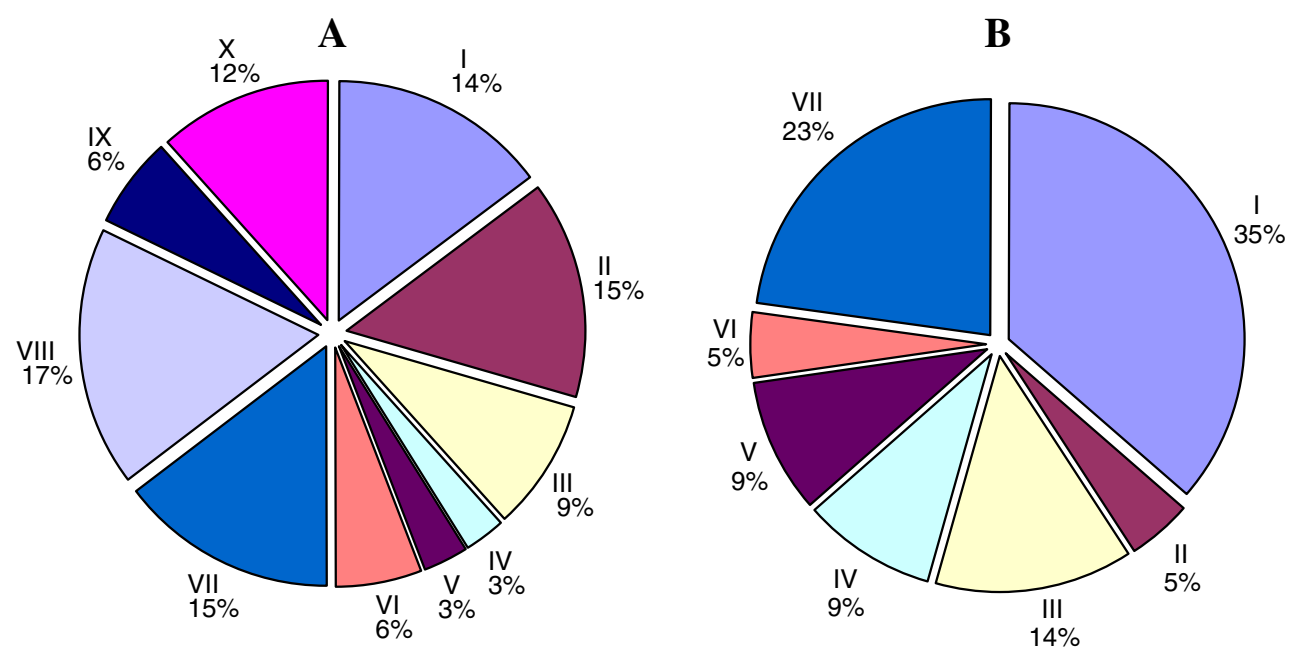

Fig. 2. Functional categories of the 56 DEPs. A. Down-regulated proteins in pituitary adenoma (34): I. neuro-endocrine and hormones; II. cytokine and cellular signal-related proteins; III. cellular defense and stress resistance; IV. mRNA splicing, transport, or translation-related enzymes; V. DNA-binding proteins; VI. Metabolic enzymes; VII. Immunologic regulation proteins and tumor-related antigen; VIII. Transport proteins; IX. cell proliferation, differentiation, and apoptosis-related proteins; X. others. B. Up-regulated proteins in pituitary adenomas (22): I. metabolic enzyme-related proteins; II. energy metabolism; III. cellular signal proteins; IV. cell cycle, cell growth and proliferation proteins; V. cellular defense response; VI. protein folding-related protein; and VII. other (taken from [4])

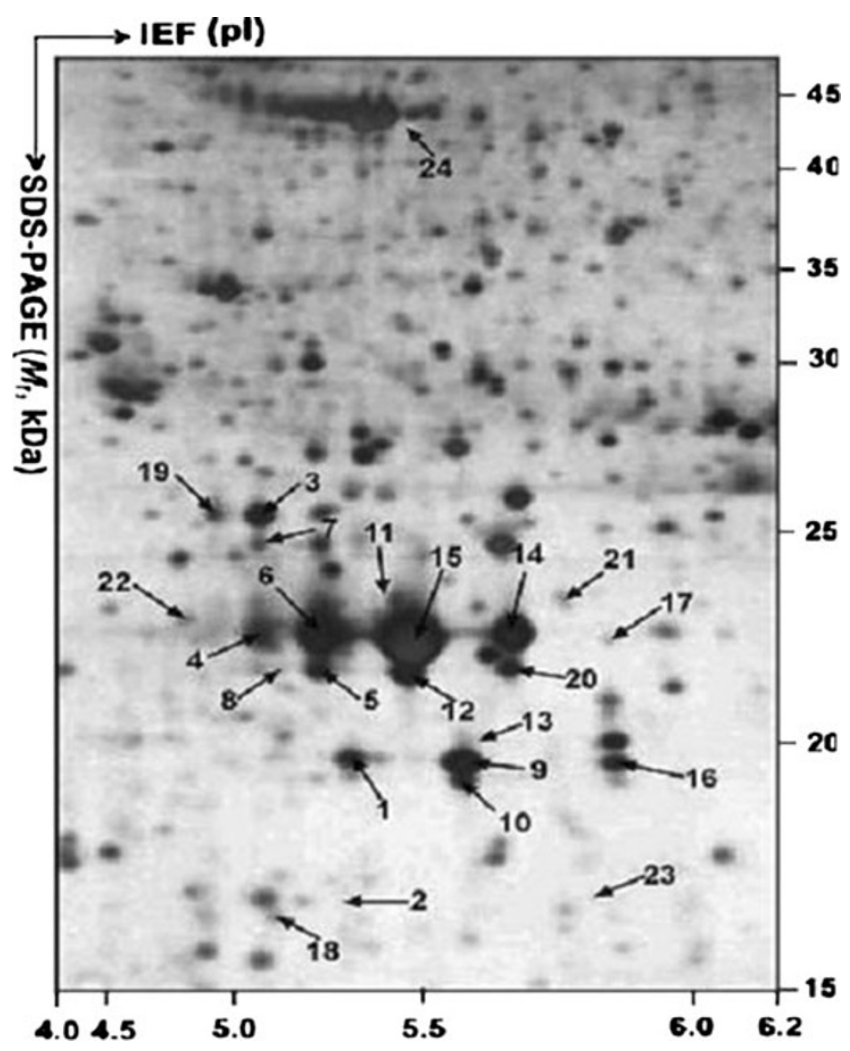

Fig. 3. xMS-characterized GH isoforms in a 2-DE map of human pituitary. IEF was performed with an IPG dry strip $(18 \mathrm{~cm}, \mathrm{pH} 3-10$ nonlinear). SDS-PAGE was performed with a polyacrylamide $(12 \%)$ resolving gel. The protein in each labeled spot was MS-characterized (taken from [5]) 


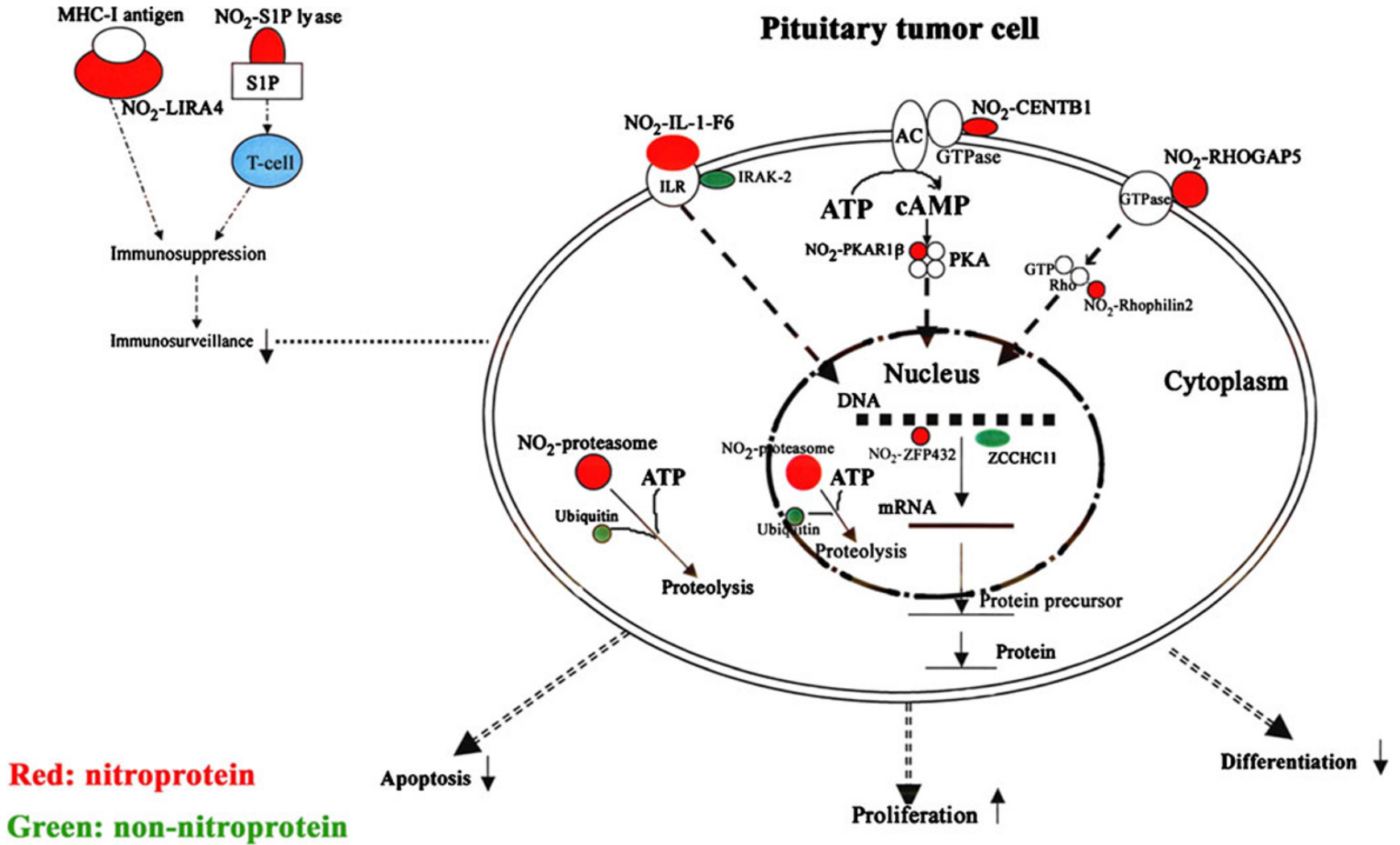

Fig. 4. Model of nitroproteins and their functions in human nonfunctional pituitary adenomas (taken from [6])
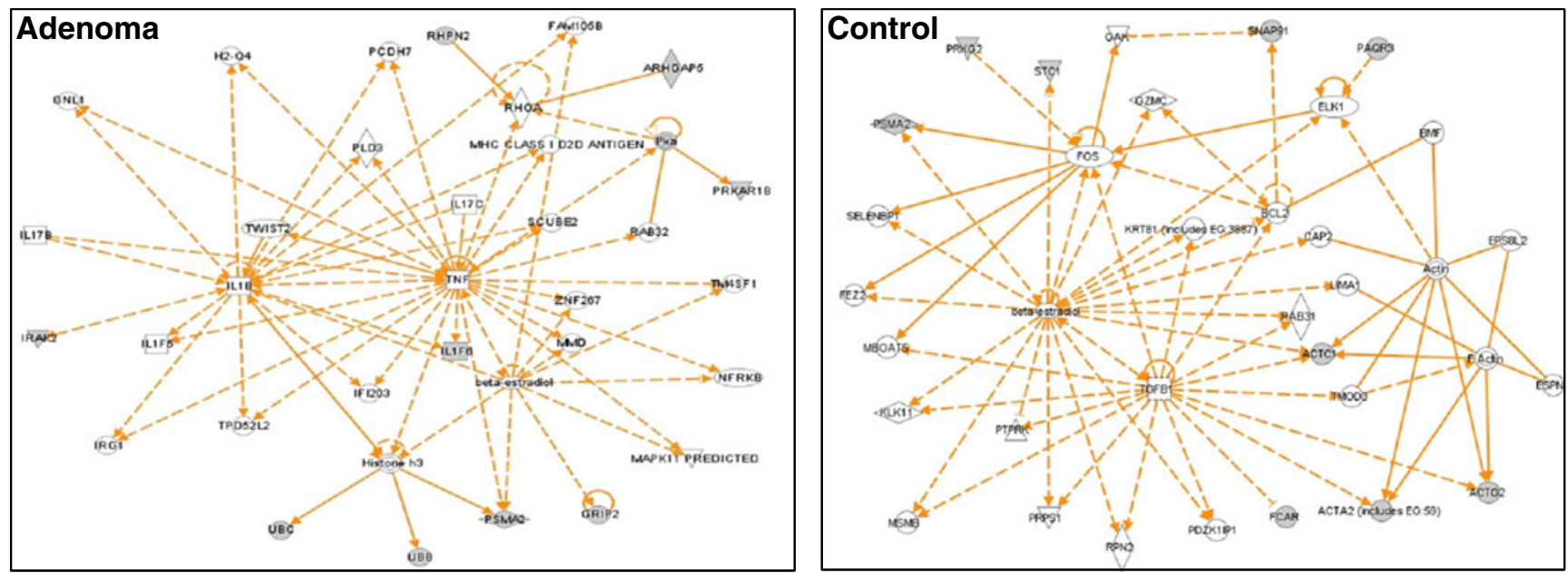

Note:

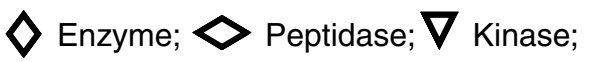

$\Delta$ Phosphatase;

Cytokine or regulator;

Others.

Fig. 5. Signaling pathway networks of nitroproteins. Network (left) derives from adenoma nitroproteomic data, and functions in cancer, cell cycle, reproductive-system disease. A gray node denotes an identified nitroprotein, or a protein that interacts with nitroproteins. Network (right) derives from control nitroproteomic data, and functions in gene expression, cellular development, and connective-tissue development and function. A gray node denotes an identified nitroprotein. An orange solid-edge denotes a direct relationship between two nodes (molecules: proteins; genes). An orange nonsolid-edge denotes an indirect relationship between two nodes (molecules: proteins; genes). The various shapes of nodes denote the different functions. A curved line means intracellular translocation; a curved arrow means extracellular translocation (taken from [9]) 
DEVELOPMENT OF A PILOT PROGRAM FOR PERSONALIZED MEDICINE FOR MALIGNANT TUMORS OF THE LIVER

Berliner $L^{1}$, Lemke $H U^{2}$, vanSonnenberg $E^{3}$, Ashamalla $H^{1}$, Dosik $D^{1}$, Gardezi $S^{1}$, Cleary $K^{4}$, Esposito $G^{5}$, Morrison $P^{6}$

${ }^{1}$ New York Methodist Hosp., Brooklyn, NY; Weill Medical

College of Cornell University, USA

${ }^{2}$ Technical University of Berlin; Berlin (D); University of

Southern California, Los Angeles, USA

${ }^{3}$ Kern/UCLA Medical Center, Bakersfield, CA, USA

${ }^{4}$ Children's National Medical Center, Washington, DC, USA

${ }^{5}$ Georgetown Univ. Medical Center, Washington, DC, USA

${ }^{6}$ Brigham and Women's Hosp., Boston, MA, USA

A program is underway to design and implement a liver tumor treatment program to allow transition from traditional medical practice toward personalized, predictive and preventative medicine (PPPM). The assessment, management and treatment of patients with malignant tumors of the liver (MTL), within the context of Model-Guided Therapy (MGT), Patient-Specific Modeling (PSM) and PPPM are addressed. It is our intention to lay the groundwork and to describe guidelines so that institutions preparing similar programs will be able to transition to MGT and to participate in a multi-center, multi-national consortium for implementing MGT.

When a patient with MTL is in the care of a treating physician, attention to their personalized needs is of paramount importance. Physicians learn to recognize the individual's emotional and spiritual needs and desires, and their physical strengths and weaknesses, which influence how that physician will form a treatment plan, based on an assessment and prediction of how that patient will respond within a given therapeutic regimen. Despite the effort spent in considering these personalized attributes, due to the lack of organized programs with specific databases to collect and analyze this data, little information is available to the treating physician for use in predicting a single individual's survival rate or treatment outcome, when a therapeutic regimen is offered. As a result, the ability to pursue personalized medicine is drastically limited.

It is believed that programs can be developed to provide personalized healthcare. The organization of such a program must be able to address the immediate and long term needs of a patient with (MTL) in an efficient, compassionate and comprehensive manner. Once a potential MTL has been detected, patients can be thought of as being within various stages of an MTL Continuum: (1) at risk; (2) MTL diagnosed and potentially curable; (3) treated and patient considered cured; (4) MTL treated and in remission; (5)
MTL treated with recurrence or progression of disease; (6) patient requiring treatment for complications and/or palliative care; and, (7) advanced MTL requiring end-of-life care. The patient, at various times during the course of their disease and treatment, will be found to progress and/or regress through the various stages of this Continuum. Therefore, a flexible algorithm needs to be developed to assist in the guidance of patients through these stages.

Programs for personalized healthcare must record patient attributes, clinical observations, and therapeutic responses in databases specifically developed for these purposes. Comprehensive information will be necessary for statistical analysis of individual patient characteristics and responses to therapeutic regimens, and to allow validation of personalized healthcare delivery and to provide the raw data for the development and testing of a comprehensive PSM. It is further postulated that the statistical analysis of an increasing number of PSM's will provide the basis for Model-Based Medical Evidence (MBME), which may provide a solid foundation for PPPM.

The program covers: (1) Diagnostic criteria and treatment algorithms; (2) Medical treatment; (3) Surgical treatment; (4) Interventional Radiology; (5) Radiation Oncology; (6) Database Development.

THE GLUTATHIONE-S-TRANSFERASE (GST) P1 313G IS A RISK ALLELE FOR THE DEVELOPMENT OF GRADE $\geq 2$ NEUROTOXICITY IN PATIENTS TREATED WITH OXALIPLATIN

Del Re $M^{1}$, Schirripa $M^{2}$, Loupakis $F^{2}$, Silvestris $N^{3}$, Colucci $G^{3}$, Falcone $A^{2}$, Danesi $R^{1}$

${ }^{1}$ Division of Pharmacology, Department of Internal Medicine, University of Pisa, Italy

${ }^{2}$ Division of Oncology, Department of Oncology, Transplants and Advanced Technologies in Medicine, University of Pisa, Italy

${ }^{3}$ Medical Oncology Department, National Cancer Institute “Giovanni Paolo II”, Bari, Italy

Background: Oxaliplatin is frequently used in the treatment of colorectal cancer in combination regimens with fluoropyrimidines. Severe neurotoxicity is the dose-limiting adverse reaction in patients receiving oxaliplatin and a potential reason for treatment discontinuation. The main metabolic route of oxaliplatin detoxification is the conjugation with glutathione by the enzyme glutathione-Stransferase (GST) P1 subclass. GSTP1 displays a polymorphism at codon $105(313 \mathrm{~A}>\mathrm{G}$ or Ile105Val $)$ with high prevalence: Ile/Ile (45-50\%), Ile/Val (42-46\%) and Val/Val (9-11\%) (Kweekel et al. 2005). The 313G allele encodes a variant GSTP1 protein with a lower enzymatic activity for 
the conjugation of various cytotoxic drugs as compared to the wild type allele (Watson et al. 1998). Previous studies have investigated the association of GTSP1 Ile105Val polymorphism with oxaliplatin toxicity, but the results of these studies were controversial and the role of the GSTP1 as a biomarker of susceptibility to develop neurotoxicity is not clear.

Aim: The aim of this study was to investigate the association of GSTP1 polymorphism Ile105Val with oxaliplatin-related neurotoxicity in advanced colorectal cancer patients.

Materials and methods: Germline DNA was extracted from 27 colorectal cancer patients treated with capecitabineoxaliplatin and suffering from neurotoxicity of at least grade $1 ; 10 / 27$ patients were affected by neuropathy grade $2-3(37 \%)$. Toxicity was graded using WHO criteria and GSTP1 313A $>\mathrm{G}$ was determined by Real-Time PCR.

Results: Genotype frequency was $11 / 27$ (40.7\%) for homozygous wild-type 313AA and 16/27 (59.3\%) for heterozygous $313 \mathrm{AG}$. No subjects showed the homozygous variant genotype. There was no significant prevalence of a specific genotype in these subjects; however, in the subgroup of patients with neuropathy grade $\geq 2$ there was a higher prevalence of the heterozygous $313 \mathrm{AG}(70 \%)$ over the wild-type $313 \mathrm{AA}(30 \%)$.

Conclusions: The results of this study suggest that the $313 \mathrm{G}$ is a risk allele and carriers of the GSTP1 $313 \mathrm{AG}$ were more prone to suffer from grade $\geq 2$ neurotoxicity than carriers of wild type GSTP1 313AA genotype. The small number of patients in this study needs to be extended in additional prospective studies, but the present finding suggest that a pharmacogenetic approach may be an important tool for optimizing chemotherapy in patients with colorectal cancer treated with oxaliplatin.

\section{References:}

Kweekel DM, Gelderblom H, Guchelaar HJ. Pharmacology of oxaliplatin and the use of pharmacogenomics to individualize therapy. Cancer Treat Rev 2005;31:90-105

Watson MA, Stewart RK, Smith GB et al. Human glutathione Stransferase P1 polymorphisms: relationship to lung tissue enzyme activity and population frequency distribution. Carcinogenesis 1998;19:275-80

\section{BREAST CANCER RISK ASSESSMENT: CON- STRUCTION OF DIAGNOSTIC WINDOWS}

Yeghiazaryan $K^{1,3}$, Cebioglu $M^{1,3}$, Braun $M^{2,3}$, Kuhn $W^{2,3}$, Schild $H H^{1,3}$, Golubnitschaja $O^{1,3}$

${ }^{1}$ Department of Radiology, University of Bonn, Germany

${ }^{2}$ Department of Obstetrics and Gynecology, University of Bonn, Germany

${ }^{3}$ Breast Center, Rheinische Friedrich-Wilhelms-University of Bonn, Germany
Why early and predictive diagnostics is crucial for long-term outcomes of breast cancer?

Breast cancer is the most common cause of cancer-related death among women with an average incidence rate of 10 12 per 100 women. Advanced stages of breast cancer lead to development of metastasis predominantly in lymph nodes, bone, lung, skin, brain, and liver. In 2005, breast cancer led to 502,000 deaths comprising $7 \%$ of cancerrelated deaths and almost $1 \%$ of all deaths worldwide. Currently applied diagnostic approaches are frequently unable to recognize early stages in tumor development that impair the outcome. At present breast-MRI is the most sensitive diagnostic tool for breast imaging; however, its specificity is limited resulting in a negative impact for surgical management in approximately $9 \%$ of cases. Early diagnosis has been demonstrated to be highly beneficial for significantly enhanced therapy efficiency and possibly full recovery.

Development of non-invasive subcellular imaging and molecular diagnostic approaches for breast cancer risk assessment

Individualized treatment algorithms and paradigm change from a late interventional approach to predictive diagnostics followed by the targeted prevention of a disease before it manifests, presents an innovative concept for advanced healthcare that is costs effective. Particularly attractive are non-invasive diagnostic approaches considering diseasespecific alterations in molecular patterns of blood cells and serum in predisposed individuals before clinically disease onset.

Group-characteristic patterns: Construction of diagnostic windows

The central idea of diagnostic windows is to create a reliable tool that allows for

- Predictive selection of cancer-predisposed individuals

- Early cancer diagnosis

- Identification of individuals at low risk of breast cancer development, so-called negative prediction.

Considering the comet-fraction patterns characteristic for individual groups of comparison, the construction of diagnostic windows for breast cancer risk assessment is proposed (Fig. 1) 


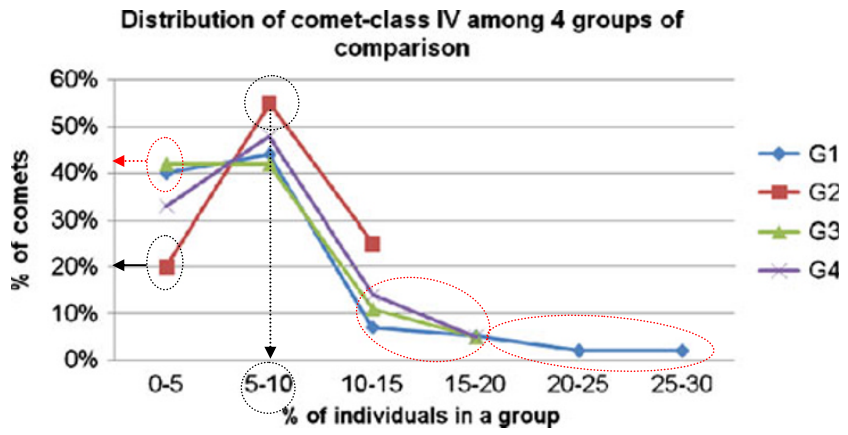

Fig. 1. demonstrates statistically significant diversities in comet-class IV (apoptotic DNA) patterns among four groups of comparison (G1 and G2 correspond to pre- and post-menopausal patients with benign breast alterations, and correspond to G3 and G4 pre- and post-menopausal patients with breast malignancies, respectively). The group-characteristic windows are well-distinguishable. Remarkable similarities between both patient groups 3 and 4 can be seen and potentially used as the pathologycharacteristic windows (red-marked zones). Noteworthy, the distribution patterns of the group of risk 1 demonstrates intermediate values between group of risk 2 on one side, and patient groups on the other side. The group of risk 1 demonstrates also an abundant number of individuals with extremely high apoptotic level. Data taken from [1]

The diagrams in Fig. 2 summarize a potential predictive power of the constructed diagnostic windows. Diagram (A) shows the frequency (\%) of patients for each of 4 groups of comparison that have been monitored for corresponding contingent of the comet-class $\mathrm{I}$ in one of the diagnostic windows. The same- for comet class IV in the diagram (C). In contrast, diagrams (B) and (D) demonstrate the frequency (\%) in corresponding windows for combined $(\mathrm{G} 1+\mathrm{G} 2$ versus $\mathrm{G} 3+\mathrm{G} 4)$ patient groups. Obviously, the diagnostic windows with the comet-class IV patterns can be effective only, when the hormonal status (pre- and post-menopausal) is considered as one of the selection parameters for subgrouping the patients and concomitant utilizing of the approach proposed by this study.

Pathology-specific comet-patterns as innovative concept of breast cancer risk assessment: Construction of diagnostic windows

The add-value of this study is the innovative concept of the qualification of pathology-specific comet-patterns. The achieved results have fully supported our hypothesis: some of the comet-patterns are highly specific for patients at high risk, whilst other diagnostic windows are highly specific for individuals at low risk. Whereas predictive diagnostic windows for patients at high risk are important to recognize the pathology before it manifests, the diagnostic windows
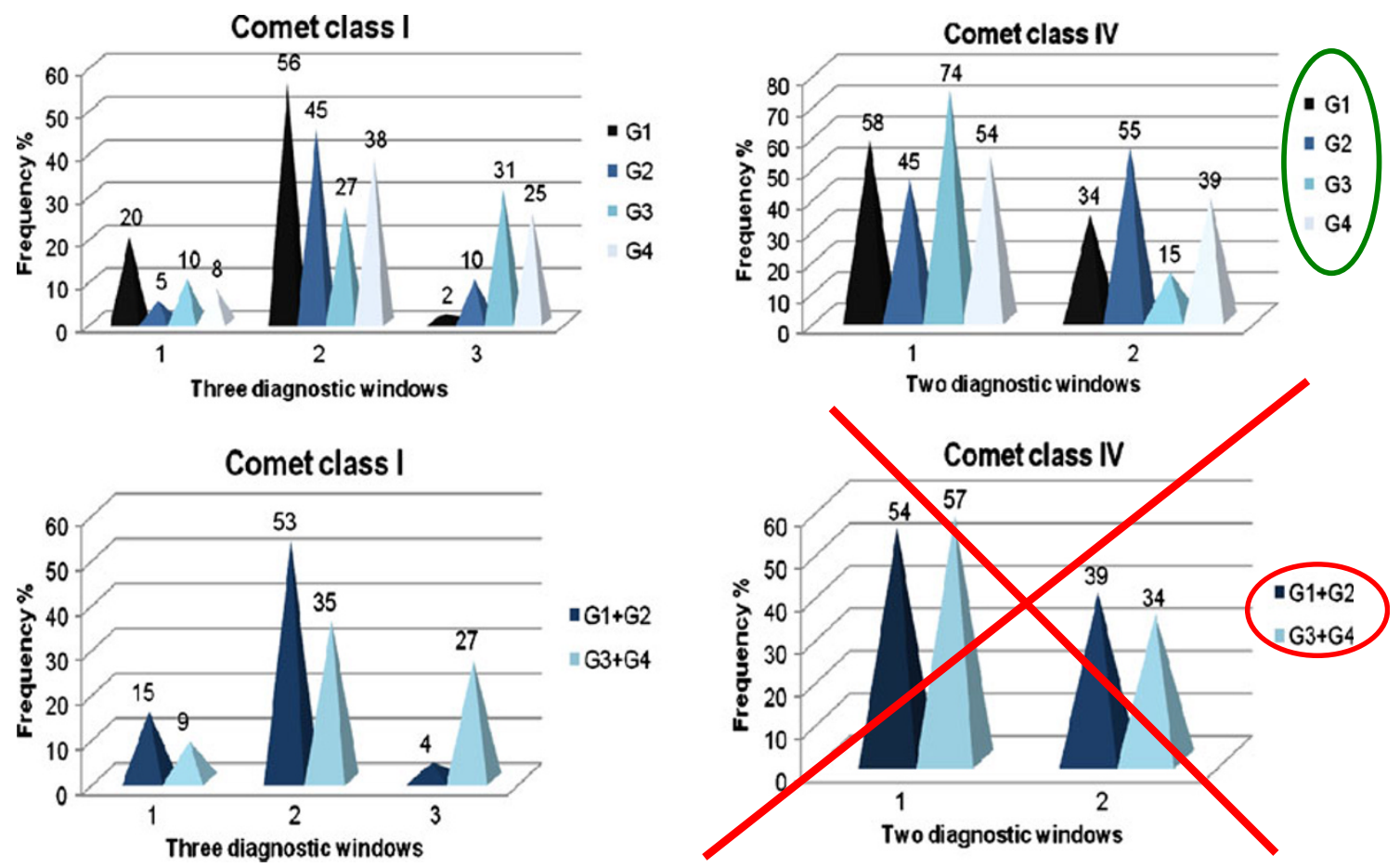

Fig. 2. Diagrams estimating a predictive power of the constructed diagnostic windows; data taken from [1] 
for individuals at low risk should help to avoid undesirable treatments and invasive approaches in the case of nonpredisposed individuals. This negative prediction is not less important compared to a positive predictive diagnosis in terms of promoting the individualized / correct patient treatments.

Reference:

1. Yeghiazaryan K, Cebioglu M, Braun M, Kuhn W, Schild HH, Golubnitschaja O. Noninvasive subcellular imaging in breast cancer risk assessment: construction of diagnostic windows. Per Med. 2011; 8: 321-30.

\section{BIOMARKER DISCOVERY: CHALLENGES AND OPPORTUNITIES}

Swanton $C$

Translational Cancer Therapeutics Laboratory, CR-UK London Research Institute, 44 Lincoln's Inn Fields, London, UK

Despite the wealth of published biomarkers in the literature, recently estimated at over 150,000, approximately 100 are in routine clinical use. Predictive biomarker identification in cancer medicine in order to identify patients with drug sensitive disease improves the health economic costs of drug therapy. Importantly, evidence suggests that successful identification of a predictive biomarker can minimise the use of ineffective therapy in the biomarker negative patient cohort. Associative learning approaches to predictive biomarker discovery are subject to data overfitting and have not resulted in the implementation of clinically useful biomarkers predictive of drug response. Functional genomics identification of drug sensitivity mechanisms using RNA interference may support novel biomarker discovery approaches. The EU framework 7 funded PREDICT consortium is taking a novel approach through the implementation of pre-surgical clinical trials for tumour genomics analysis with parallel laboratory functional genomics screens to identify the tumour and stromal genomics basis for drug response. Caveats to this approach and tumour biological mechanisms impeding the implementation of personalised medicine will be discussed.

PREDICTIVE AND PROGNOSTIC MEDICINE: THE ROLE OF KRAS AND BRAF MUTATIONS AS BIOMARKERS IN COLORECTAL CANCER

Hagan $S^{1}$, Doyle $B^{2}$

${ }^{1}$ Department of Vision Sciences, Glasgow Caledonian University, Glasgow G4 0BA, Scotland

${ }^{2}$ Centre for Colorectal Diseases, St. Vincent's University Hospital, Dublin 4, Ireland
In developed countries, colorectal cancer (CRC) is the 3rd most common malignancy and 2nd most frequent cause of cancerrelated death. It therefore offers numerous challenges to clinicians in treatment regime decision-making. A recent genome-wide analysis of tumours showed that 20-100 protein-encoding genes were mutated. It is thought that a small number of these genes may be critical in driving cancer progression. However, many cancer-causing mutations are subtle missense mutations that cannot be easily detected by conventional protein analysis (western blotting, ELISA). KRAS (and also BRAF) is one of the most commonly mutated cancer genes, but no single antibody is available that can reliably differentiate normal from mutated versions of the protein. Understanding the mutational status of these genes may provide prognostic information in CRC. More importantly, it has it appears that KRAS and BRAF mutations predict response to anti-EGFR monoclonal antibody therapy. As EGFR can also signal via the PI3-Kinase pathway, there is considerable interest in the potential roles of members of this pathway (such as PI3Kinase and PTEN) in predicting treatment response. Therefore, new techniques that allow identification of these "biomarkers" will aid in the diagnosis of cancer, help in treatment decision making and may also serve as future therapeutic targets.

EARLY CANCER DIAGNOSIS AND NOVEL THERAPEUTIC OPTIONS RESULTING FROM SYSTEMATIC PROTEOME PROFILING OF TUMOURASSOCIATED STROMA CELLS

Paulitschke $V^{1}$, Haudek-Prinz $V^{2}$, Slany $A^{2}$, Mohr $T^{2}$, Griss $J^{2}$, Gerner $C^{2}$

${ }^{1}$ Department of Dermatology, Medical University of Vienna, Währinger Gürtel 18-20, 1090 Vienna, Austria

${ }^{2}$ Department of Medicine I, Medical University of Vienna, Borschkegasse 8a, 1090 Vienna, Austria

The discovery of novel biomarkers is essential for the improvement of virtually all therapies as well as the early detection of malicious diseases. Nevertheless, most biomarkers discovered so far have not satisfied the hopes laid upon them.

In contrast to most other proteomics research groups we do not only focus on blood serum and whole tissue samples but have started to systematically investigate sub-cellular fractions of different cell types in different functional states creating a "protein-atlas" of primary human cells. We believe that only if we know what is "normal" for a cell in different functional states we will be able to deduct specific pathological changes that qualify as biomarkers.

Over the last 6 years we have performed over 10,000 nanoLC-MS/MS experiments studying 70 distinct cell types, 
isolated from 16 different tissues in 81 different functional states identifying a total of 10,340 distinct proteins. A focus of this work was the systematic investigation of the contribution of non-parenchymal stroma cells to tumour promotion and the relation of these processes to inflammation. Therefore we established proteome maps of various tumour cells, normal as well as tumour-associated fibroblasts and endothelial cells from bone marrow, colon, lung, liver and skin. Furthermore, we investigated well-defined functional cell states by treating cells in vitro with IL-1beta or TGF-beta, respectively. Indeed, some proteome alterations observed in tumor-associated cells were related to inflammation and wound healing activities. Intriguingly, some features of tumour-associated stroma cells appeared to be common irrespective of the individual donor and tissue of origin and unrelated to the proteome signatures of inflammation or wound-healing. This pointed to general mechanisms of tumour-stroma interactions which may be recognised and specifically targeted by appropriate therapeutic interventions. Furthermore, this aberrant cell phenotype was accompanied by the secretion of a characteristic set of proteins which may serve as biomarkers. Currently we are establishing sensitive and robust quantification strategies for the assessment of these biomarkers in blood serum. We have started a clinical study of melanoma patients for clinical validation of our results.

\section{PREDICTION AND PROGNOSIS: IMPACT OF GENE EXPRESSION PROFILING IN PERSONALIZED TREATMENT OF BREAST CANCER PATIENTS}

Mallmann $M R^{1,2}$, Staratschek-Jox $A^{2}$, Rudlowski $C^{1}$, Braun $M^{1}$, Gaarz $A^{2}$, Wolfgarten $M^{1}$, Kuhn $W^{1}$, Schultze $J L^{2}$ ${ }^{1}$ Department of Obstetrics \& Gynecology, Center for Integrated Oncology, University Hospital Bonn, SigmundFreud-Strasse 25, D-53105 Bonn, Germany

${ }^{2}$ LIMES (Life and Medical Sciences Bonn), University Bonn, Carl-Troll-Str. 31, D-53115 Bonn, Germany

Breast cancer is the most common cancer of women and a complex disease, whose heterogeneity is increasingly recognized. Despite considerable improvement in breast cancer treatment and survival, a significant proportion of patients seems to be over- or undertreated with current therapy. To date, single clinicopathological parameters show limited success in predicting the likelihood of survival or response to endocrine therapy and chemotherapy. Consequently, new gene expression based prognostic and predictive tests are emerging that promise an improvement in predicting survival and therapy response. Initial evidence has emerged that this leads to allocation of fewer patients into high-risk groups allowing a reduction of chemotherapy treatment. Moreover, pattern-based approaches have also been developed to predict response to endocrine therapy or particular chemotherapy regimens. Whereas most of these approaches are at the stage of translational research, several are ready to be implemented into clinical practice. Irrespective of current pitfalls such as lack of validation and standardization, these pattern-based biomarkers accelerate identifying novel molecular targets and will prove useful for clinical decision making in the near future, especially if more patients get access to this form of personalized medicine.

\section{VNTR AT HER2/NEU GENE AMPLIFICATION BORDER IN BREAST CANCER-THE "WEAKEST LINK" IN GENE AMPLIFICATION?}

Kovalenko SP, Matsenko NY

Institute of Molecular Biology and Biophysics, 630117, Novosibirsk, Russia

Overexpression and amplification of the HER-2/neu gene is observed in $20-30 \%$ of breast cancer cases and predicts more frequent relapse and shorter survival time. The initial events and molecular mechanisms of amplification in the region around $H E R 2 / n e u$ as well as the precise boundaries of amplified regions remain to be obscure. This study was aimed to define the boundaries of amplified chromosome 17q12-q21 region around HER2/neu gene. Structural peculiarities of amplification boundary regions were investigated by various techniques.

Gene dosage analysis was performed for several genes located around HER2/neu gene in 154 breast cancer tissue samples. The copy number of genes around Her $2 /$ neu, namely ppparbp, znfnla3 Casc3 and top $2 a$ genes were analyzed by comparative TaqMan real time PCR. Fifty six samples (36\%) were found to be her2/neu-amplified $\left(N_{\text {HER } 2 / n e u} \geq 1,5\right)$, the boundaries for HER2-containing amplicons were mapped.

Observed results suggest that similar positions of amplified region boundaries in different breast cancer samples are not accidental and may be related with the DNA structural and/or sequence peculiarities of boundary regions. Trying to find "special" sequences at the amplicon boundaries, we performed the analysis of sequence flexibility in the region TBC1D3HER2/neu - TOP $2 A$ using the TwistFlex program. The analysis revealed several high flexible sequences in SOCS7, FBX047, HER2/neu and ZNFN1A3 genes. The locations of amplified region boundaries in $30 \%$ of cases were inside ZNFN1A3 gene. Organization of DNA sequence and nucleotide composition ofFlexZNF-1 and FlexZNF-2 were similar to AT-rich islands of common fragile site (FS). Sequences of 
FlexZNF-1 and FlexZNF-2 have strong potential to form secondary structures according to the analysis by Mfold software (GCG Wisconsin Package ${ }^{\mathrm{TM}}$ ).

Thus, the analysis of DNA sequence in TBC1D3- HER2/ $n e u-T O P 2 A$ region revealed the existence of two flexible sequences in theZNFN1A3 gene sequence with the strong potential to form stable secondary structures which may affect normal DNA replication at these sites and result in DNA double strand break appearance. The location of FlexZNF-1 and FlexZNF-2 strongly coincided with the location of amplified around HER2/neu gene fragment boundaries observed by real time PCR analysis.

This observation indicates involvement of Flex sites in the process of intrachromosomal DNA amplification. Since FlexZNF-1 and FlexZNF-2 sites are mainly composed from tandem repeats, the variability of repeats numbers in the regions was analyzed in the local population. At least two allelic variants were found, so amount of repeats is genetically predetermined feature. We assume that the length of the Flex site on the border of HER2/neu amplificon can be considered as the essential point in the process of intrachromosomal amplification around HER2/neu gene.

To summarize the results of the investigation:

- The borders for HER2/neu amplification were precisely localized at least in $30 \%$ cases;

- The borders of amplification were shown to contain repetitive highly flexible sequences;

- The number of repetitive sequences at the borders of HER2/neu amplification varies in population.

PREDICTIVE VALUE OF DOMINANT NODULE IN HASHIMOTO THYROIDITIS. A CLUE FOR A PERSONALIZED APPROACH FOR GREATER RISK OF PAPILLARY THYROID CARCINOMA

Trovato G, Amico P, Martines GF, Castaing M, Torrisi A, Vecchio GM, Salvatorelli L, Catalano D, Magro G

Department G.F. Ingrassia, Anatomic Pathology, and Department of Internal Medicine, Diagnostica e Terapia Medica, "University of Catania, Catania, Italy

Hashimoto's Thyroiditis (HT) or chronic lymphocytic thyroiditis is an autoimmune disease in which the thyroid gland is gradually destroyed by a variety of cell- and antibody-mediated immune processes. HT can undergo morphological changes over the time, resulting in at least three different histological variants (classic, nodular or fibrotic variant). Interestingly some patients with HT may develop one or more nodules larger than $>1 \mathrm{~cm}$, so-called dominant nodules (DNs). Due to the association between HT and papillary thyroid carcinoma (PTC), the detection of DNs is alarming, prompting physicians to perform fine needle aspiration cytology (FNAC) to rule out malignancy. The incidence, nature (benign vs. malignant) and clinical impact of DN in HT is still controversial.

The aim of the study is to investigate: (1) the clinicopathological features of DN in HT; (2) the prevalence of DNs that are actually PTC; (3) the predictive value of DN for a concurrent PTC occurring elsewhere in the thyroid gland.

Patients and methods: We reviewed 230 consecutive cases of thyroidectomy (partial or complete), age $48.44 \pm 11.05$ years (range 24-80) with a clinical and histologically proven diagnosis of HT. Histological revision of all hematoxylin/ eosin (H\&E) stained sections was performed. Any nodule, grossly reported (original pathology report) and histologically confirmed, with a diameter $\geq 1.5 \mathrm{~cm}$, merging from the surrounding thyroid parenchyma, was conventionally defined as a $\mathrm{DN}$.
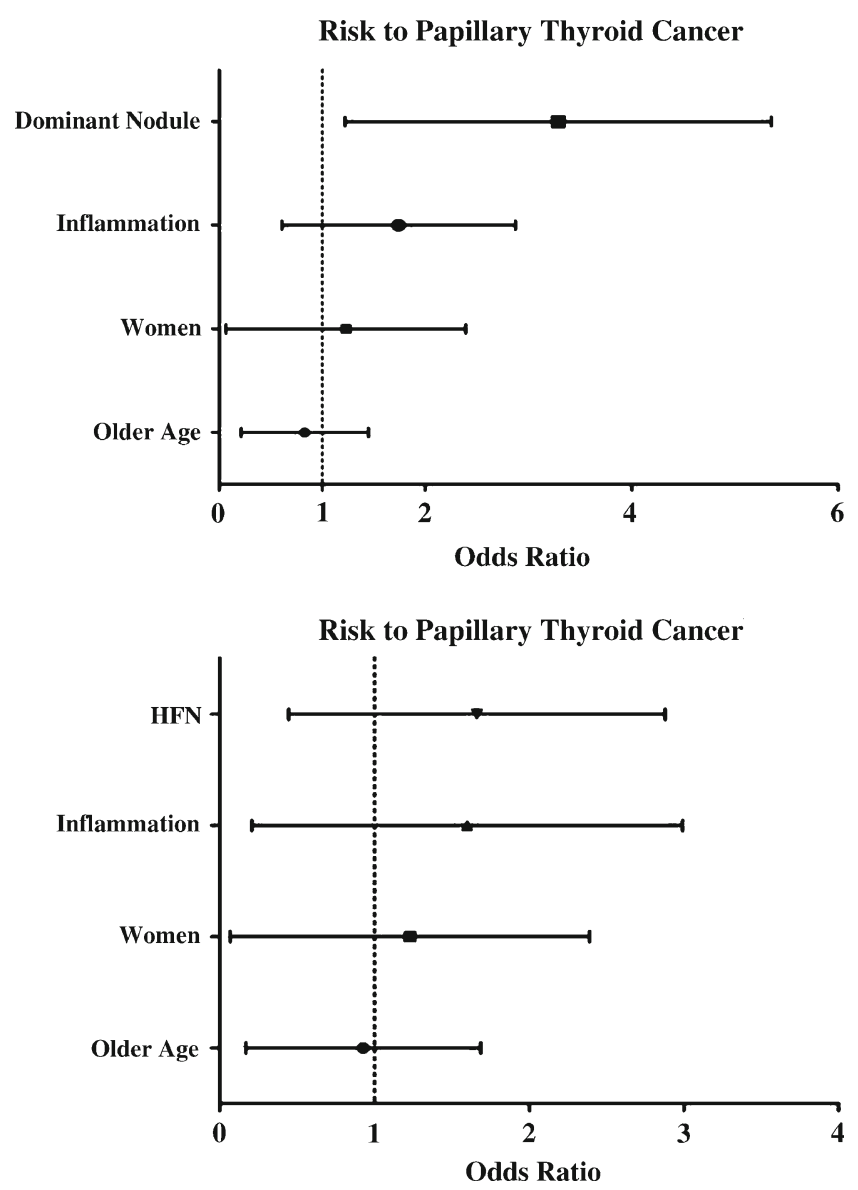
Results: Among the 230 cases of HT 69 showed at least one DN ( $\geq 1.5 \mathrm{~cm})$. Histologically most of DNs $(57 / 69,82.6 \%)$ where benign lesions, i.e. hyperplastic follicular nodules (HFNs), while only 12 DNs (17.4\%) were PTC. HFNs were composed of Hurthle or non-Hurthle cells, with or without association with chronic inflammation.

PTC was present in other part of the thyroid gland in $17 / 57$ $(29.8 \%)$ of the patients with a benign HFN-DN, and in 18 / $161(11.2 \%)$ patients with HT but without any DN.

The Odds-ratio to PTC showed a four-fold increased risk to PTC when the thyroid exhibits a DN, and a lower risk in older patients.

Also excluding DN that were actually PTCs, the Odds-ratio showed an increased risk to PTC for patients with a HFN- DN By MLR, the absolute dimension of the nodule is predictive of malignancy. This model, moreover, predicts $18.2 \%$ of the variance to the dimension of PTC.

Table. Multiple Linear Regressions to PTC dimensions (cm)

$$
\begin{array}{llllll}
R & R^{2} & F & \text { sig } & \beta & p
\end{array}
$$$$
\begin{array}{llll}
0,427 & 0,182 & 16,690 & <\mathbf{0 , 0 0 0 1}
\end{array}
$$

Age, $y$

$-0,099 \quad 0,103$

Inflammation

$0,173 \quad \mathbf{0 , 0 0 8}$

DN dimensions,

$0,443<\mathbf{0 , 0 0 0 1}$

$\mathrm{cm}$

\section{PTC: Papillary Thyroid Carcinoma; DN: Dominant Nodule $(\geq 1,5 \mathrm{~cm})$}

Comments

1) Only a minority of DNs in HT are actually PTCs. Most DNs are hyperplastic follicular lesions that could be clinically managed, avoiding unnecessary surgery.

2) Nonetheless, the presence of a DN in HT, even if benign, increases four-fold the risk of PTC. A seemingly protective effect is associated with an older age in these patients.

3) Also HFN-DNs, i.e. lesions that conceivably should have been assessed benign at FNAC, increase, even at a lower extent, the risk for PTC elsewhere in the thyroid gland.

Conclusion: A great care in the use of imaging information as predictive tools for PTC in large DNs of HT is warranted.

\section{IDENTIFICATION OF CALPONIN-H2 AS POTEN- TIAL SERUM-BASED MARKER FOR THE EARLY DIAGNOSIS OF BREAST CANCER}

Debald $M^{1}$, Franken $S^{2}$, Heukamp $L C^{3}$, Linke $A^{4}$, Wolfgarten $M^{1}$, Walgenbach $K J^{1}$, Rudlowski $C^{1}$, Holdenrieder $S^{4}$, Braun $M^{1}$, Hartmann $G^{4}$, Kuhn $W^{1}$, Walgenbach-Brünagel $G^{4}$
${ }^{1}$ Department of Obstetrics and Gynecology, Centre for Integrated Oncology, University of Bonn, Germany

${ }^{2}$ Institute for Biochemistry and Molecular Biology, University of Bonn, Bonn, Germany

${ }^{3}$ Institute of Pathology, Centre for Integrated Oncology, University of Bonn, Germany

${ }^{4}$ Institute for Clinical Chemistry and Clinical Pharmacology, Centre for Integrated Oncology, University of Bonn, Germany

Purpose: To date, clinical breast examination, imaging by mammography as well as tumor biopsy are the only recommended methods for breast cancer screening, while mainly clinicopathological parameters determine the adjuvant therapy of this disease. In order to implement a personalized management of breast cancer patients, reliable biomarkers are urgently needed to improve the early detection or provide evidence of the prognosis for each individual patient through expression levels in tumor tissue or body fluids.

Methods: The nuclear matrix composition of human breast cancer tissue $(n=14)$, benign control tissues $(n=2)$ and healthy control tissues $(n=2)$ was analyzed by highresolution two-dimensional gel electrophoresis and mass spectrometry. The identified proteins were validated by onedimensional immunoblot in an individual sample set. A sandwich ELISA was used to determine the serum levels of calponin-h2 in breast cancer patients $(n=11)$ and healthy controls $(n=5)$.

Results: In this setting, we identified five proteins that were upregulated in human breast cancer tissue (14/14), but absent in any of the four healthy and benign control tissues $(p=0,0003)$. These spots were also present in the investigated human breast cancer cell lines outlining the epithelial character of the identified proteins. One of these breast cancer specific proteins has been confirmed to be Calponinh2 by mass spectrometry and one-dimensional immunoblot in an individual sample set of three additional breast breast cancer tissues and two additional healthy control tissues. Compared to healthy controls, serum levels of calponin-h2 were significantly increased in patients with early breast cancer $(p=0,022)$.

Conclusion: In an effort towards personalized and predictive medicine in breast cancer, Calponin h2 has been shown to be a potential serum-based marker for the early diagnosis of this disease. Further studies are ongoing to validate these promising results in a larger sample set.

\section{PHARMACOGENOMICS IN CANCER THERAPY}

González Neira A

Spanish National Cancer Research Centre (CNIO), Madrid, Spain; EPMA-Spain 
Although the current cancer therapies are improving rapidly, it is often hindered by drug resistance and treatment-related toxicities. Interindividual and interethnic variability in drug pharmacokinetics and pharmacodynamics may be explained by commonly occurring genetic polymorphisms in drug-metabolizing enzymes and drug transporters. Although to date pharmacogenomics studies have been focused on a reduced number of genes, the development of the new technologies are allowing us to focus these studies on the integration of multiple drug pathways and also to perform analysis at the genome-wide level. These new approaches allow a more comprehensive analysis of genetic factors influencing drug efficacy and toxicity in breast cancer and could reveal possible unknown mechanisms involved in drug response.

\section{TUMOUR PROGRESSION PROGNOSIS AND ENGINEERING OF INDIVIDUALISED CLINICAL TREATMENTS FOR MALIGNANT GLIOMAS}

Trog D, Yeghiazaryan K, Schild HH, Golubnitschaja $O$

Department of Radiology, Rheinische Friedrich-WilhelmsUniversity of Bonn, Germany

Malignant gliomas are highly lethal cancer disease. Thus, median survival of 14.6 and 12.1 months was demonstrated when either chemoradiation or radiation alone had been applied. Irrespective of the treatment, glioblastoma patients have been shown to benefit from epigenetic silencing of MGMT (O6-methylguanine-DNA-methyltransferase) expression, DNA-repair activity of which was compromised by promoter methylation (an independent favourable prognostic factor associated with longer survival in patients with glioblastoma). The fact that those patients whose tumours are not methylated at the MGMT-promoter do not benefit from temozolomide (TMZ) treatment, suggests a predictive role of MGMT methylation status in therapy response towards TMZ-treatment. For these patients, alternative treatment approaches should be applied. However, under TMZ-treatment significant differences in survival (range of 17 to 30 months) have been demonstrated even for patients whose tumours were methylated at the MGMT-promoter. Depending on the patient, individual data measured in the primary human glioblastoma cells showed a wide range of response to TMZ treatment in vitro. This finding calls for further predictors of individual therapeutic responses in treated glioblastomas. Therefore, molecular mechanisms of therapy resistance are of particular interest in malignant glioblastomas with a hypermethylated MGMT-promoter.

Engineering of individualised clinical treatments for malignant gliomas guided by individual molecular profiling is particularly relevant for the creation of improved postoperative treatment of peripheral tumour regions, the diffuse character and disseminated growth of which make the treatment of malignant gliomas especially complicated. It has been clearly documented that current post-operative therapeutic approaches may favour peripheral tumour progression and select for a more aggressive relapse than before these treatments were introduced as the standard care. Consequently, the specific expression patterns forcing pro-invasive and pro-angiogenic activity of malignant cells were found to be characteristic particularly for the treated tumour periphery as simulated by the above described algorithms. These results strongly support the clinical observations of increased aggressiveness and relatively poor response to second line therapies in post-operative, chemoradiation-treated, malignant gliomas at the time of relapse. In addition to the methylation status of MGMTpromoter, tumour patterns combining expression levels of the pathology-specific genes reported might be useful for the prediction of individual therapy response and disease progression in malignant glioblastomas with a hypermethylated MGMT-promoter. Individualised treatment algorithms can be designed using established gene expression patterns applied to primary cell cultures obtained from individual patients. Individual drug-toxicity and response to anticancer therapy can be estimated.

General progress in treatment of brain tumour patients is expected by introducing directed therapy approaches which would specifically suppress the activity of molecular targets of glioma invasion in order to control invasive cell subpopulations escaping surgical removal and lethal radiation exposure.

\section{TOWARDS A PERSONALIZED PARADIGM OF EXPERIMENTAL ANTICANCER RESEARCH WITH "3S" CONCEPT}

Moiseeva EV, Semushina SG, Bojenko VK

Institute of Bioorganic Chemistry, Moscow, Russian Federation

Earlier, we developed three novel inbred mouse strains (RB-strains, carriers of $\mathrm{Rb}(8.17) 1 \mathrm{Iem}$ Robertsonian translocation). They exhibited various but high incidences of spontaneous mammary cancer (MC) rarely associated with ovary/uterus cancer and/or leukaemia/lymphoma. New classification of mouse $\mathrm{MC}$ has been worked out to disclose similarities with human breast cancer (BC) pathology. Our way to follow each laboratory mouse during its life-span individually (as a patient) and original approaches to test anticancer drugs (ACD) have been developed during last two decades [1]. This direction towards a personalised 
paradigm of experimental research has been currently resulted in " $3 \mathrm{~S}$ "concept:

The 1st S: Set of non-SPF mouse strains as an adequate model of familial aggregation of human breast cancer with multiple cancer cases and leukaemia/lymphoma; The 2nd S: Steps of experimental research in vitro (cancer and immune cells separately) and in vivo: transplanted (preliminary tests to discover proper dosage, application time, et cetera), spontaneous (therapeutic application in females with spontaneous $\mathrm{MC}$ with/without tumour extirpation), and precancerous (preventive regimen in females before spontaneous MC occurrence);

The 3rd S: Subgrouping approach to disclose beneficial and non-beneficial subgroups in both experimental and control groups concurrently and to show both positive and negative effects of $A C D$, respectively.

Several experimental areas of our research should be mentioned to illustrate our concept validity in relation to personalized/predictive/preventive medicine (" $3 \mathrm{P}$ " medicine).

First of all, adequacy of our mouse models was approved by histopathology (evident similarity of some mouse mammary carcinoma types to corresponding types of human BC). Furthermore, apparent analogies in recurrence rate and survival in relation to the stage of mouse $\mathrm{MC}$ to corresponding parameters for human $\mathrm{BC}$ patients were revealed after surgery. Moreover, we found similar predictive values of particular histopathologic patterns of extirpated murine MC samples to related patterns of human BC. In both species, "comedo" mammary carcinoma patterns predict early recurrence and/or poor survival; and medullar carcinoma is characterized by extensive leukocyte infiltration and unexpectedly good outcome.

Moving further to "3P" conception of modern medicine, we showed that some laboratory blood and serum parameters (i) predicted subclinical period of murine MC (time before transplanted tumour manifestation); (ii) correlated with a stage of tumour growth in transplanted models; and (iii) allowed to forecast time of spontaneous MC detection in precancerous females of RB-strains. Additionally, some laboratory parameters (related to anaemia and chronic inflammation in the murine liver/kidney/lung/hart) were associated with beneficial or non-beneficial effects of immunotherapy by local interleukin-2.

Importantly, non-SPF mice (especially, aged animals) show chronic inflammation in many organs similarly to the majority of human BC patients. For instance, viral hepatitis does interfere with treatment procedures in both species. These data may (i) imply failure of SPF mouse models to reproduce concurrent pathologies in human $\mathrm{BC}$ patients and (ii) approve non-SPF ones as appropriate mouse models for preclinical ACD testing.

In summary, we showed utility of " $3 \mathrm{~S}$ " concept on the way to personalized experimental anticancer research.

Reference:

1. Moiseeva E. Anti-breast cancer drug testing. Original approaches. Novel set of mouse models. Lambert academic publishing, 2009

\section{CANCER-ASSOCIATED IMMUNE-MEDIATED SYNDROMES: PATHOGENIC VALUES AND CLINICAL IMPLEMENTATION}

Merkulova $A M^{1}$, Kostalevskaya $A V^{1}$, Suchkov $S V^{1,2}$

${ }^{1}$ I.M. Sechenov First Moscow State Medical University, Moscow, Russia

${ }^{2}$ Moscow State Medical Dentistry University, Moscow, Russia

The ability of tumors to provoke formation of cancerassociated secondary immunodeficiency (CASID) with predominant suppression of CMI and cancer-associated secondary immunodeficiency with clinical autoimmunity syndrome (CASICAS) with triggering of a set of the autoimmune deviations is appearing to be a key event in the restriction of hosts' anti-tumor immunity. In order to reach a point where immunological phenotypes in GBM and BCC can be clarified clinically and, partly, pathogenically, we have conducted a series of studies of typical and atypical types of immune responsiveness in patients with GBM (Glioblastoma multiforme) and BCC (Basal cell carcinoma). For GBM and BCC three scenarios of the involvement of the immune responsiveness have been established in a series of our studies, i.e., (i) malignancy with no immunopathology, (ii) malignancy as CASID, and (iii) malignancy as CASICAS. All of those scenarios demonstrated significant differences in their immune-mediated manifestations which, in turn, were proven to reveal close associative relationships with a specific clinicopathologic type and clinical manifestations of the tumor. CASID and CASICAS share two common features, i. e., (i) signs of immunodeficiency and (ii) a tandem of the deviations within the adaptive and innate links of the host immune responsiveness. At the same time, CASID and CASICAS are distinct pathogenically and clinically, and in terms of depth of the immune deviations observed, CASID patients manifest a breakage in both links, whereas in CASICAS patients, a breakage in the adaptive link would dominate. To get these differences clarified, we summarized major types of the immune imbalances and sets of clinical and clinicopathologic manifestations to illustrate the abovementioned features in CASID and CASICAS patients. There are distinct close correlations between clinicopathologic features of the disease course and sets of the immunemediated imbalances in patients harboring the tumors. The 
latter implicates a panel of the new immunodiagnostic and immunoprognostic criteria for patients with solid tumors, i.e., BCC, MCC and GB, which is of great value for clinical practice.

In particular, the blood levels of some of the immunocompetent cells, state of their functional activity, serum titers of the antigenic markers and autoantibodies, apoptotic parameters, and others may be accepted as additional and clinically informative criteria to be implemented for immunological monitoring and immunotherapy of patients with solid tumors.

\section{PROGNOSTIC SIGNIFICANCE OF MIR-21 AND MIR-143 EXPRESSION IN TISSUE SAMPLES OF COLORECTAL CARCINOMA AND COLORECTAL LIVER METASTASES}

Pešta $M^{1}$, Topolčan $O^{1}$, Kulda $V^{2}$, Řehoř $L^{2}$, Svaton̆ $M^{2}$, Holubec $L^{1}$, Černý $R^{2}$, Liška $V^{3}$

${ }^{1}$ Department of Internal Medicine II, Faculty of Medicine in Pilsen, Charles University in Prague, Czech Republic

${ }^{2}$ Department of Biochemistry, Faculty of Medicine in Pilsen, Charles University in Prague, Czech Republic

${ }^{3}$ Department of Surgery, University Hospital and Medical Faculty in Pilsen, Czech Republic

MicroRNAs, which are endogenously expressed regulatory noncoding RNAs, have an altered expression in colorectal cancer.

The aim of our study was to assess a potential link between miR-21 and miR-143 expression and the prognostic / clinical / pathological features of colorectal carcinoma (CRC) and colorectal liver metastases (CLM).

Methods: The estimation was performed in 46 paired (tumour and control) tissue samples of CRC. We studied 30 tissue samples of CLM. miR-21 and miR-143 expressions were quantified using the method of RT qPCR.

Results: A correlation between miR-21 and miR-143 expression and a disease-free interval (DFI) (Wilcoxon; $P=0.0026$ and $P=0.0191$, respectively) was identified. DFI was shorter in patients with a higher expression of both miR-21 and miR-143, which is a putative tumour suppressor. There was a higher expression of miR-21 and lower expression of miR-143 in CRC tissue in comparison with adjacent normal colon tissue $(P<0.0001 ; P<0.0001$, respectively). Similarly, we observed a higher expression of miR-21 and a lower expression of miR-143 in CLM in comparison with normal colon tissue $(P<0.0001$; $P<0.0001$, respectively).

Conclusion: Our results support the hypothesis about oncogenic / tumour-related function of miR-21 and show its association with DFI. The role of miR-143 in carcino- genesis seems to be more complex than it has been expected.

This study was supported by grants NS 10240-3/09 and Nr. 10230 from the Ministry of Health of the Czech Republic.

\section{VITAMIN D AND CANCER DISEASES}

Fuchsova R, Topolcan O, Pazdiora P, Svobodova S, Vrzalova J, Krakorova G, Spisakova M, Treska V, Treskova I, Pesek M, Finek $J$

Faculty Hospital and Medical Faculty in Pilsen, Charles University, Czech Republic

Vitamin D is a fat-soluble steroid prohormone which plays a major role in the calcium and phosphorus homeostasis. It is known that most cells express Vitamin D receptor and about $3 \%$ of the human genome is directly or indirectly regulated by Vitamin D. However, 40 to $75 \%$ of the world's population is Vitamin D deficient. Vitamin D levels have been associated with many disorders, diseases and related outcomes: osteoporosis, cancer, cardiac risk, autoimmune and reumatoid diseases, transplant patients, ect. The aim of our study was to determine the frequency of vitamin D hypovitaminosis in patients with different malignant cancer diseases.

Methods: Serum levels of 25-OH vitamin D were measured using ECLIA immunoassay manufactured by Roche in 215 healthy individuals and in 170 patients with colorectal, lung, prostate and breast (pre- and postmenopausal) cancer. Serum levels in cancer patients were compared to healthy group and further correlated with disease stage within each diagnosis.

Results: Vitamin D levels were found to be significantly lower in cancer patients compare to healthy group. $39 \%$ of all cancer patients show values below $47,5 \mathrm{nmol} / \mathrm{L}-$ cutoffs set as 5 th percentile of healthy group. $29 \%$ of patients with colorectal cancer, $35 \%$ patients with breast cancer, $64 \%$ of patients with lung cancer and $18 \%$ of patients with prostate cancer had levels of D vitamin below cut off value. Authors have confirmed the correlation between disease stage and serum levels in patients with colorectal lung and premenopausal breast cancer. No correlation was found for postmenopausal breast cancer and for prostate cancer.

Conclusion: Authors found high incidence rate of severe hypovitaminosis D in cancer patients among Czech population. This rate is significantly higher when comparing literature data. Correlation with a disease stage was found.

Support: This study was supported by VZ and grants NS9727-4, NS9998-4, NT11017-5. 
MULTIPLEX PANEL FOR OVARIAN CARCINOMA Vrzalova $J^{1,3}$, Topolcan $O^{1,3}$, Novotny $Z^{2}$, Presl $J^{2}$, Pesta $M^{3}$ ${ }^{1}$ Immunoanalytic Laboratory, Dep. of Nuclear Medicine, Faculty Hospital in Pilsen, Czech Republic

${ }^{2}$ Dep. of Gyneacology, Faculty Hospital in Pilsen, Czech Republic

${ }^{3}$ Central Isotopic laboratory, Fac. of Medicine in Pilsen, Charles University in Prague, Czech Republic

Aim: As a continuation of our pilot study [1] commercially available xMAP multiplex panel specifically designed for ovarian cancer is to be tested in larger patient cohorts. The multiplex panel includes Macrophage Migration Inhibitory Factor (MIF), prolactin (PRL), CA-125, leptin, osteopontin (OPN) and IGF-II.

Methods:

\section{Cancer groups:}

- Fifty patients with ovarian carcinoma

- Seven patients with border line tumour of ovaria (BTO)

\section{Benign groups:}

- Thirty patients with benign ovarian cysts (BOC)

- Thirty patients with endometriosis

- Eleven patients with cardiac insufficiency

Serum levels of multiplex markers were measured by Beadlyte ${ }^{\circledR}$ Human Cancer Biomarker Panel from Millipore (USA) and Luminex 100 instrument (Luminex, USA). Simultaneously, levels of CA125, TK, TPS, HE4 and Monototal were measured by routine immune analytical methods.

Results: From the multiplex markers, the best ROC characteristics were observed for CA125, IGFII and OPN. HE4 marker had the best ROC characteristic from all measured markers for differentiation of ovarian carcinoma and $\mathrm{BOC}$ or endometriosis. In the multiplex panel, significant differences in marker levels between ovarian carcinoma and $\mathrm{BCO}$ were founded for CA125, OPN and IGFII (lower in carcinoma) and between ovarian carcinoma and endometriosis for IGFII, OPN, PRL (for both higher in carcinoma). For OPN, MIF and HE4 the levels were different in BTO compare to BCO. CA125 measured either by multiplex or routine method was higher in endometriosis compare to benign cysts. Using multi-parametric data handling ROC curves for multiplex panel (AUC 0.87636) were comparable to CA125 alone to distinguish between ovarian carcinoma and $\mathrm{BOC}$.
Conclusion and prospective: Multiplex may enable the use of a scoring system for clinical use in distinguishing between several pathologies. However, the choice of markers used for the panel is crucial.

Support: Study was supported by the research grant project NS10258-3.

Reference:

1. Vrzalova J, Prazakova M, Novotny Z, Topolcan O, Casova M, Holubec L Jr. Test of ovarian cancer multiplex xMAP technology panel. Anticancer Res. 2009; 29: 573-6.

\section{PPPM EARLY IN LIFE: MOLECULAR AND EPIDEMI- OLOGICAL MODELS FROM PAEDIATRIC CANCER}

Rovigatti $U$

Department of Oncology, University of Pisa Medical School, Via Roma 55, 56127 Pisa, Italy

In his 2008 Nobel lecture, Harald zur Hausen spends only a few words on the association of high-risk HPVs with cervical cancers, although for this discovery his Nobel Prize was awarded. Instead, his emphasis is placed on additional and new situations of infectious agents as triggers, especially of childhood cancers [1]. Infections, which are being suspected to trigger a higher proportion of paediatric malignancies, could become a new and important Paradigm of PPPM Early in Life.

My group has tackled this problem for several years, starting from seminal observations on cancer clusters, i.e.: space-time associations of new diagnoses, which have been extensively studied epidemiologically, mostly in the UK and US. The work by L. Kinlen has suggested that they could be explained by population-mixing effects, recognized now in several cancer-clusters [2]. Isolated groups, for example in rural communities, are unexposed and therefore susceptible to a still-to-define infectious agent (probably a virus $=\mathrm{X}$-virus), carried along my immigrations of newcomers. Immigrants typically originated from more densely inhabited areas: they were exposed to the XAgent and therefore protected by "herd-immunity". The isolated communities lack such herd immunity and are susceptible to the cancer-inducing X-agent [2]. A slightly different model was proposed by M. Greaves, taking into account the molecular biology of paediatric cancers and especially leukaemia [3]. Monozygous twins display sometimes $(10 \%$ of cases) a genetically identical disease (identical chromosomal translocation breakpoints): this could be only explained by metastatic-diffusion of cancer cells from one twin to the other through placental anastomoses. Interestingly, disease could be diagnosed in twins up to 9 years apart. This led to discovering that 
chromosomal translocations are already present at birth (Guthrie's cards), although it takes variable time to "express them" into overt disease [3]. Even more puzzling, such translocations are present also in the normal population, although at a lower level (in approx. 50\% for the CML breakpoint and in up to $100 \%$ normal individuals for MLL aberrations) [4]. Greaves model requires two distinct events, but could be reconciled with Kinlen's population-mixing if the $\mathrm{X}$-virus corresponded to the later trigger. We initially studied a cancer-cluster of Neuroblastoma (NB) cases in Southern Louisiana and serendipitously discovered a cytoplasmic Virus: Micro-Foci inducing Virus (MFV) [5]. A similar virus was isolated from paediatric lymphomas. Experimental evidence link these viruses to childhood carcinogenesis, because MFVs: 1 . induce malignant transformation in vitro; 2. induce the appearance of specific chromosomal (DNA) aberrations; 3. cause tumorigenesis in experimental animals. [6]. The possibility that MFV is the X-Virus or 2nd trigger implied by Kinlen/Greaves studies is being investigated.

Other experiments link MFVs to the Fatigue Syndromes often associated to malignancies (CFS/CRF). Recently, the Retrovirus XMRV was linked to CFS, but this is artefact. MFV shares the evidence for CFS/CRF trigger including high induction of RNASEL [7].

In conclusion our MFV model, in association with Kinlen and Greaves, could provide a more general explanation for a portion of childhood cancers. In view of the unique characterization of both MFV genotypes and chromosomal aberrations (fingerprints), this could become an excellent PARADIGM of PPPM Early in Life.

\section{References:}

1. Zur Hausen, H. The search for infectious causes of human cancers: where and why. Virology. 2009 Sep 15;392(1):1-10.

2. Kinlen, L. Childhood leukaemia, nuclear sites, and population mixing. Br J Cancer. 2011; 104: 12-18.

3. Greaves, M. Infection, immune responses and the aetiology of childhood leukaemia. Nat Rev Cancer. 2006;6:193-203.

4. Bäsecke J, Griesinger F, Trümper L, Brittinger G. Leukemia- and lymphoma-associated genetic aberrations in healthy individuals. Ann Hematol. 2002;81:64-75.

5. Rovigatti U. Isolation and initial characterization of a new virus: Micro-Foci inducing virus or MFV. C R Acad Sci III. 1992;315:195-202.

6. Rovigatti U, Piccin A, Colognato R, Sordat B. Preliminary Characterization of a New Type of Viruses Isolated from Paediatric Neuroblastoma and Non-Hodgkin's Lymphoma: potential Implications for Aetiology. Intn. Conference Childhood Leukaemia. Section P1-18 pp I-IV, September 2004, London, United Kingdom, Editor: CwL.
7. Rovigatti U, Selli C, et al. "Of Mice and Men-1333 Viruses and Prostate Cancer: What Is the Next Step?". European Urology. 2010;58(5):684-686.

\section{REgULATION OF THE PHOSPHATASE, PP2A AS A CLASSIFIER OF CANCER PATIENTS WITH POTENTIAL SENSITIVITY TO THE PP2A ACTIVA- TOR, FTY720}

Grech $G$

Department of Pathology, University of Malta, Malta

The importance of feedback mechanisms involved in suppression of growth factori-nduced signals is gaining importance both to understand molecular mechanisms of disease and also as potential targets to specific therapeutics. The use of biomarkers to derive knowledge required for the classification of patients into therapeutic groups has been extensively used in various malignancies. Our studies show that deregulation of the phosphatase, $\mathrm{pp} 2 \mathrm{a}$ is central to enhanced proliferation of haematopoeitic progenitor cells at the expense of suppressed differentiation induction. Using an erythroid progenitor cellular model, we showed that differentiation can be blocked by constitutive expression of the pp2a subunit, alpha4. This was mainly through maintained activation of the mTOR pathway. The block in differentiation was rescued using the pp $2 \mathrm{a}$ activator, FTY720 in the same model. Interestingly, pp2a dephosphorylates and suppresses the activity of various signalling pathways, namely the Akt kinase. Cancer patients with suppressed phosphatase activity are potentially sensitive to the pp2a activator, FTY720. In addition to cellular models, our previous studies on patient material derived from Triple negative Breast Cancer patients show that patients can be classified using Akt phosphorylation as a biomarker. High Akt phosphorylation correlates with low BRCA1 expression, a mechanism that directly involves the phosphatase pp2a. This new class of triple negative breast cancer patients might benefit from therapy directed towards activation of pp2a.

New insights in the mechanism of pp2a regulation form the basis of potential identification of variants that affect the phosphatase activity. Further studies will allow association of these variants with activity of the phosphatase and identification of biomarkers to screen for such variation. Characterisation of the mechanisms of pp2a inhibition will allow classification of patients into therapeutic groups sensitive to pp2a activators. 